\title{
On a Theorem of Hernández and Nashed
}

\author{
M. Dörfner
}

\begin{abstract}
We prove a theorem on the global invertibility of nonlinear maps in metric vector spaces. As corollaries our result yields a theorem of Hernández and Nashed, as well as the classical Banach-Mazur lemma and an invertibility criterion by Browder.
\end{abstract}

Keywords: Global invertibility, local invertibility, proper maps, ray-proper maps, closed maps AMS subject classiflcation: $47 \mathrm{H} \mathrm{15,26} \mathrm{B} \mathrm{10,54} \mathrm{C} \mathrm{10,} \mathrm{58} \mathrm{C} 07$

Throughout this note, $X$ and $Y$ are real metric vector spaces, and $\Phi: X \rightarrow Y$ is a continuous (in general, nonlinear) operator satisfying (without loss of generality) $\Phi(0)=$ 0 . Recall that such an operator is called proper if the pre-image $\Phi^{-}(C)$ of any compact set $C \subset Y$ is compact. Our starting point is the classical Banach-Mazur lemma which may be stated as follows:

Theorem 1 (see [2]). A continuous operator $\Phi$ is a global homeomorphism if and only if $\Phi$ is a local homeomorphism and proper.

Loosely speaking, this theorem shows that properness is the "missing" property if an operator is only locally invertible, but not globally invertible. Results of this type are not only of theoretical interest, but also important in view of many applications. Thus, to prove the global invertibility of a proper map simply reduces to proving its local invertibility which may often be achieved by quite elementary means (e.g., by the inverse function theorem and its various generalizations, see [5] for example).

The standard first-year calculus example of a local homeomorphism which is not globally invertible is

$$
\Phi\left(x_{1}, x_{2}\right)=\left(e^{x_{1}} \cos x_{2}-1, e^{x_{1}} \sin x_{2}\right) \quad\left(\left(x_{1}, x_{2}\right) \in \mathbb{R}^{2}\right) .
$$

Of course, the map (1) is not proper since $\Phi^{-}([-2,0] \times[-1,1]) \supset\{0\} \times \mathbb{R}$. Moreover, this map is not closed either since $\Phi\left(\mathbb{R}^{2}\right)=\mathbb{R}^{2} \backslash\{(-1,0)\}$. This is not accidental, by the following

Theorem 2 (see [4]). A continuous operator $\Phi$ is a global homeomorphism if and only if $\Phi$ is a local homeomorphism and closed.

A further result in this spirit was recently given by J. E. Hernández and $M$. Z. Nashed [7]. A continuous operator $\Phi: X \rightarrow Y$ is called ray-proper if the pre-image

M. Dörfner: Berliner Allee 69, D - 63225 Langen 
$\Phi^{-}([\theta, y])$ of the "ray" $[\theta, y]=\{t y: 0 \leq t \leq 1\}$ is compact for any $y \in Y$. Obviously, every proper map is ray-proper but not vice versa. Moreover, one can show that an operator $\Phi$ is proper if and only if $\Phi$ is ray-proper and closed. Indeed, this easily follows from the well known fact that an operator $\Phi$ is proper if and only $\Phi$ is closed and $\Phi^{-}(\{y\})$ is compact for every $y \in Y$ (see [3] for example).

Theorem 3 (see [7]). A continuous operator $\Phi$ is a global homeomorphism if and only if $\Phi$ is a local homeomorphism and ray-proper.

The trivial example $X=\mathbb{R}$ and $\Phi(x)=\arctan x$ shows that the requirement of ray-properness of $\Phi$ cannot be weakened to "point-properness" (i.e. the requirement that $\Phi^{-}(\{y\})$ be compact for all $\left.y \in Y\right)$.

We illustrate Theorem 3 by a simple but typical example. Let $X=Y=C([0,1])$, equipped with the usual max-norm, and let $\Phi$ be the linear operator defined by

$$
\Phi(x)(t)=\int_{0}^{t} x(s) d s
$$

The range of $\Phi$ is the subspace $C_{0}^{1}([0,1])=\left\{y \in C^{1}([0,1]): y(0)=0\right\}$, and $\Phi$ is invertible on this subspace with inverse $\Phi^{-1}(y)=y^{\prime}$. However, $\Phi^{-1}$ is not bounded, since we have equipped $C_{0}^{1}([0,1])$ with the $C$-norm. It is easy to check that

$$
\Phi^{-}([\theta, y])= \begin{cases}{\left[\theta, y^{\prime}\right]} & \text { if } y \in C_{0}^{1}([0,1]) \\ \{\theta\} & \text { if } y \notin C_{0}^{1}([0,1])\end{cases}
$$

and hence $\Phi$ is ray-proper. On the other hand, $\Phi$ is neither proper (since $\Phi$ is a compact operator) nor closed (since $C_{0}^{1}([0,1])$ is not closed in $\left.C([0,1])\right)$. Thus, in this case Theorems 1 and 2 do not apply, but Theorem 3 does. In fact, $\Phi$ cannot be a local homeomorphism, by Theorem 3.

Now we are going to prove another Banach-Mazur type result which yields all the preceding theorems as corollaries.

Theorem 4. A continuous operator $\Phi: X \rightarrow Y$ is a global homeomorphism if and only if $\Phi$ satisfies the following two conditions:

(a) $\Phi$ is a local homeomorphism.

(b) For each $y \in Y$ there is a continuous map $x:[0,1] \rightarrow X$ such that $x(0)=\theta$ and $\Phi(x(t))=t y$ for $0 \leq t \leq 1$.

Proof. The necessity of conditions (a) and (b) is clear. To prove the sufficiency, suppose that $\Phi$ satisfies conditions (a) and (b); we have to show that $\Phi$ is onto and one-to-one. Given $y \in Y$, choose $x:[0,1] \rightarrow Y$ according to condition (b). Since $y=\Phi(x(1))$ we see that $\Phi$ is onto. Moreover, since $\Phi$ is locally homeomorphic the map $x$ is also uniquely determined. Now put $x_{1}:=x(1)$ and let $x_{2}$ be another point in $X$ with $\Phi\left(x_{2}\right)=y$. Putting $z(t):=t\left(x_{2}-x_{1}\right)+x_{1}$ and $y(t):=\Phi(z(t)) \quad(0 \leq t \leq 1)$ by condition (a) we conclude that $y(t) \quad(0 \leq t \leq 1)$ is a non-degenerate closed curve in $Y$ satisfying $y(0)=y(1)=y$. By conditions (a) and (b), for each $t \in[0,1]$ we find a unique continuous map $x_{t}:[0,1] \rightarrow Y$ satisfying $x_{t}(0)=\theta$ and $\Phi\left(x_{t}(s)\right)=s y$ for $0 \leq s \leq 1$. For $t$ sufficiently close to 1 we get $x_{t}(1)=z(t)$, by condition (a), hence $x_{1}(1)=z(1)=x_{2}$. But $\Phi\left(x_{1}(s)\right)=s y$ for all $s \in[0,1]$, and therefore $x_{1}(s) \equiv x(s)$, by the uniqueness of $x$. In particular, we conclude that $x_{2}=x_{1}(1)=x(1)=x_{1}$ which shows that $\Phi$ is injective 
To illustrate Theorem 4 we give two examples. Consider again the operator (1). As we already remarked, $(-1,0)$ is not in the range of $\Phi$ and thus condition (b) of Theorem 4 fails.

Now let the operator $\Phi: \mathbb{R}^{2} \rightarrow \mathbb{R}$ be defined by

$$
\Phi\left(x_{1}, x_{2}\right)=x_{1} \cos x_{2} \quad\left(\left(x_{1}, x_{2}\right) \in \mathbb{R}^{2}\right) .
$$

Since $\Phi^{-}(\{0\})$ is unbounded $\Phi$ is not proper and thus not ray-proper, too. Let $A:=$ $\{1\} \times\left\{2 \pi n+\frac{1}{n} \mid n \in \mathbb{N}\right\}$. Since $\Phi(A)$ is not closed $\Phi$ cannot be a closed operator. However, $\Phi$ satisfies condition (b) of Theorem 4. Indeed, for $y \in \mathbb{R}$ choose $x(t)=$ $(t y, 0) \quad(0 \leq t \leq 1)$. One easily checks that $\Phi$ is not a local homeomorphism and thus condition (a) of Theorem 4 is not fulfilled.

Now we show that both Theorem 2 and Theorem 3 (and hence Theorem 1, too) follow from our Theorem 4.

\section{Theorem 5. Theorem 4 implies Theorem 2.}

Proof. We have to show that every closed local homeomorphism satisfies condition (b). If $y=\theta$, we simply choose $x(t) \equiv 0$. Let $y \neq \theta$. Since $\Phi$ is a local homeomorphism with $\Phi(\theta)=\theta$, for some $\varepsilon>0$ we can find a unique continuous map $x:[0, \varepsilon) \rightarrow X$ satisfying $x(0)=\theta$ and $\Phi(x(t))=t y$. Let $\tau \in(0,1]$ be the supremum of all possible $\varepsilon$ with this property, and let $\left(t_{n}\right)_{n}$ be any sequence in $[0, \tau)$ converging to $\tau$. We claim that $\left(x\left(t_{n}\right)\right)_{n}$ contains a convergent subsequence.

In fact, if this is false, then the set $A=\left\{x\left(t_{n}\right): n \in \mathbb{N}\right\}$ is trivially closed and hence also the set $\Phi(A)$, by assumption. Therefore we have $\tau y \in \Phi(A)$ and $\Phi\left(x\left(t_{n}\right)\right)=t_{n} y=$ $\tau y$ for some $n \in \mathbb{N}$. But $t_{n}<\tau$ for all $n$, hence $y=\theta$ contradicting our assumption on $y$.

Let $\left(t_{n_{j}}\right)$; be a subsequence of $\left(t_{n}\right)_{n}$ such that $x\left(t_{n_{j}}\right) \rightarrow x_{*}$ for some $x_{*} \in X$. Since $\Phi$ is continuous, we have $\Phi\left(x_{*}\right)=\tau y$. But the local invertibility of $\Phi$ implies then that $x$ may be defined for some $t>\tau$ if $\tau<1$. This shows that $\tau=1$, and we are done

Theorem 6. Theorem 4 implies Theorem 3.

Proof. We have to show that every ray-proper locally homeomorphic operator $\Phi$ satisfies condition (b). Given $y \in Y$, we define $x:[0, \tau) \rightarrow X$ and $\left(t_{n}\right)_{n}$ as in the proof of Theorem 5. Since $\Phi$ is ray-proper, the set $\Phi^{-}([\theta, y])$ is compact, and hence $\left(x\left(t_{n}\right)\right)_{n}$ contains a convergent subsequence. The remaining part of the proof is precisely as before

Let us remark that Theorem 4 - in contrast to the theorems of Banach-Mazur, Browder and Hernández and Nashed - is rather an injectivity result than an injectivity and surjectivity result since surjectivity is trivial by condition (b). That means that in order to apply Theorem 4 we need to know the surjectivity of the operator a priori.

We point out that other results in the spirit of Theorem 4 may be proved by means of compactness arguments. If $X$ and $Y$ are finite-dimensional, the properness of a continuous operator $\Phi: X \rightarrow Y$ simply means that $\|\Phi(x)\| \rightarrow \infty$ as $\|x\| \rightarrow \infty$; such operators are usually called coercive. In this case it is a useful device to apply just the classical Banach-Mazur lemma. On the other hand, if $X$ and $Y$ are infinite-dimensional, 
coercivity does not imply properness, and one has to impose additional conditions on $\Phi$. We give a sample result in this direction. Following [1], we denote by $\|\Phi\|_{\chi}$ the lower $\chi$-norm of the operator $\Phi$, i.e.

$$
\|\Phi\|_{\chi}=\inf \left\{\frac{\chi(\Phi(M))}{\chi(M)}: M \subset X \text { bounded, with } \chi(M)>0\right\},
$$

where

$$
\chi(M)=\inf \{\varepsilon>0: M \text { admits a finite } \varepsilon \text {-net }\}
$$

denotes the (Hausdorff) measure of non-compactness of $M$. Then we have the following

Theorem 7 (see [6: p. 25]). Suppose that $X$ and $Y$ are infinite-dimensional Banach spaces, and $\Phi: X \rightarrow Y$ is a local homeomorphism with $\|\Phi\|_{\chi}>0$. Then $\Phi$ is a global homeomorphism.

In [7: Corollaries 3.5 and 3.7) the authors give two results on the global invertibility of operators of the form $\Phi=I-K$ with $K$ being compact. Theorem 7 shows that any such operator in an infinite-dimensional Banach space is always globally invertible if it is locally invertible. In particular, the hypothesis of [7: Corollaries 3.5 and 3.7] that $\Phi^{-}([\theta, y])$ be bounded for all $y \in Y$ may be dropped.

\section{References}

[1] Akhmerov, R. R., Kamenskij, M. I., Potapov, A. S., Rodkina, A. S. and B. N. Sadovskij: Measures of Noncompactness and Condensing Operators. Basel: Birkhäuser 1992.

[2] Banach, S. and S. Mazur: Über mehrdeutige stetige Abbildungen. Studia Math. 5(1934), $174-178$.

[3] Bourbaki, N.: Elements de mathematique. Paris: Hermann 1949.

[4] Browder, F. E.: Covering spaces, fiber spaces and local homeomorphisms. Duke Math. J. 21 (1954), $329-336$.

[5] Deimling K.: Nonlinear Functional Analysis. Berlin: Springer 1985.

[6] Dörfner, M.: Beiträge zur Spektraltheorie nichtlinearer Operatoren. Ph. D. Thesis. Würzburg: Bayerische Julius-Maximilians-Universität 1997.

[7] Hernández, J. E. and M. Z. Nashed: Global invertibility in nonlinear functional analysis. In: Proc. 2nd Intern. Conf. on Fixed Point Theory Appl. (ed.: Kok-Keong Tan). Singapore: World Sci. 1991, pp. $229-247$. 